\title{
Difficulties Mastered are Opportunities Won PULMONOLOGIST'S PERSPECTIVE
}

\author{
*Sonal Kansra AND Paul Aurora \\ *Department of Pediatric Respiratory Medicine, Sheffield Children's Hospital and \\ Department of Respiratory Medicine, Great Ormond Street Hospital for Children, NHS Foundation Trust, London, UK. \\ p.aurora@ucl.ac.uk
}

$\mathrm{T}$ here has been a huge improvement in survival of cystic fibrosis $(\mathrm{CF})$ patients in the developed world over the last two decades; they are likely to survive into the fifth decade of life. There are promises of even further improvements in survival and quality of life with newer therapies that are mutationspecific. Recognition of electrolyte abnormalities in the sweat in the fifties by di Sant'Agnese, et al. [1] led to the development of the 'sweat test' to diagnose CF that continues to be the gold standard in its diagnosis. In 1989, the gene that encodes the $\mathrm{CF}$ transmembrane conductance regulator (CFTR) protein on the long (q) arm of chromosome 7, was first identified. To date, more than 1500 CFTR gene mutations with the potential to cause cystic fibrosis disease have been described. Genotype-phenotype correlations remain poor (except in pancreatic insufficiency) and there is variation in the severity of illness even in siblings carrying the same mutation profile.

Cystic fibrosis remains a clinical diagnosis with classical phenotypic features in the presence of either: a) evidence of CFTR dysfunction, mostly in the form of elevated sweat chloride; or b) demonstration of two disease-causing mutations. Mutational analysis has especially been useful in the context of newborn screening and when sweat tests are equivocal. Knowledge of the common mutations in a population is important in the diagnostic armamentarium as well as for prognosis.

Much of the progress in cystic fibrosis survival has been achieved incrementally in small steps rather than a huge leap or cure. Proactive management through specialist centers, pancreatic replacement therapy, inhaled antibiotics and mucolytics have contributed to this improvement. Newborn screening has minimized the diagnostic delay, and early nutritional treatment contributes to improved growth and cognition. Early recognition may also help to delay the onset of established lung disease although the evidence for this is mixed. Prolific research into the molecular basis of the disease and clinical trials has complemented the clinical care available to $\mathrm{CF}$ patients in developed countries.
While most treatments are 'symptomatic', development of small molecule compounds that directly target the underlying CFTR defects has the potential of being disease-modifying. Ivacaftor is the first such treatment which targets the CFTR-gating mutation Gly551Asp (previously termed G551D). Clinical trials have demonstrated benefit in terms of an increase in lung function, decreased sweat chloride, weight gain, improvement in patient-reported quality of life, and reduction in number of respiratory exacerbations with Ivacaftor [2].

As mutation-specific therapies such as Ivacaftor become available, correct genotyping of CF patients will become increasingly important for targeted therapy. In this context, Kawoosa and colleagues [3] present an intriguing clinical and genetic (mutational) description of 18 cases of cystic fibrosis from the Indian state of Jammu and Kashmir. Remarkably within a high risk group of 100 , almost a fifth of children were found to have CF based on sweat testing and mutation analysis. This relatively high proportion underscores the importance of awareness of CF in South Asian populations who were previously thought to have low prevalence of the condition.

Although a limited number of mutations were looked at in the patients with suggestive sweat tests, most patients with CF were found to have either Phe508del or $3849+10 \mathrm{~kb} \mathrm{C}>\mathrm{T}$ mutations. Both these mutations are associated with relatively severe phenotype, and patients presented with the classic symptoms of failure to thrive and pulmonary involvement (although the children with $3849+10 \mathrm{~kb} C>\mathrm{Tt}$ ended to have later onset of symptoms).

Difficulties mastered are opportunities won. Diagnosis of $\mathrm{CF}$ in developing countries is impeded by lack of awareness, poor healthcare access and financial constraints. While diagnosis of $\mathrm{CF}$ in South Asia must primarily rely on high diagnostic suspicion and availability of sweat electrolyte analysis, CFTR mutation analysis can also be complementary to an early diagnosis in these children. In addition, knowledge of the genotype provides an opportunity to benefit from genotypespecific treatment in the future. 
Funding: None; Competing interest: None stated.

\section{REFERENCES}

1. di Sant'Agnese PA, Darling RC, Perera GA, Shea E. Sweat electrolyte disturbances associated with childhood pancreatic disease. Am J Med. 1953;15:777-84.

2. Accurso FJ, Rowe SM, Clancy JP, Boyle MP, Dunitz JM,
Durie PR, et al. Effect of VX-770 in persons with cystic fibrosis and the G551D-CFTR mutation. N Engl J Med. 2010;363:1991-2003.

3. Kawoosa MS, Bhat MA, Ali W, Hafeez I, Shastri S. Clinical and mutation profile of children with cystic fibrosis in Jammu and Kashmir. Indian Pediatr. 2014;51:185-9.

\title{
CFTR Mutations in India: Need to do More! GENETICIST'S PERSPECTIVE
}

\author{
KATTA M GIRISHA \\ Division of Medical Genetics, Department of Pediatrics \\ Kasturba Medical College, Manipal University, Manipal-576 104 \\ girish.katta@manipal.edu
}

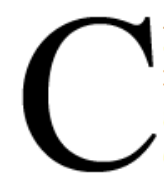
ystic fibrosis is an autosomal recessive multisystem disease with significant morbidity and mortality in all parts of the world. Contrary to the popular belief, early genetic studies on cystic fibrosis confirmed that the condition does occur in India and the most prevalent delta F508 mutation occurred frequently, in $19-56 \%$ of cases [1-3]. The largest series on mutation analysis in Indian patients found that delta F508 mutation accounted for 31.1\% mutations [4]. It was also noted that Indians may have a different spectrum of mutations as several new mutations were observed [4-6]. The carrier frequency of $0.42 \%$ appears to be an underestimate considering a 10 times higher incidence in Caucasians and the disease is not studied well in Indian population [7]. It also needs to be emphasized that it is not easy to diagnose and manage this condition in our country [8].

Kawoosa and colleagues [9] have screened a group of patients with clinical symptoms of cystic fibrosis from Jammu and Kashmir. Then they performed testing for two specific mutations known to cause cystic fibrosis in selected group of 15 patients with the diagnosis of cystic fibrosis. It is important to note that the authors have chosen to screen only for two mutations. Though this is often used as a strategy in a resource-scarce setting, given the allelic heterogeneity, it would have been better to screen the entire gene. Labeling cases with only one mutation as cystic fibrosis transmembrane conductance regulator related metabolic syndrome is also not appropriate as the entire gene was not sequenced in them. They could identify only 16 alleles in 15 patients leaving nearly half the alleles unidentified. Hence it would be premature to draw conclusions on the commonality of the mutations considering the small study group and mutation detection strategy. Nevertheless this study highlights the frequency of the condition and two selected mutations in the studied population and the need for effective strategies for mutation detection that can go a long way to offer genetic counseling services for these families.

Recently mutation-specific treatment has been tried in patients with at least one G551D mutation that was based on prior knowledge of the effect of the mutation on CFTR channel and its repair by the drug $[10,11]$. Hence it is important that such studies are expanded to provide comprehensive mutation testing facilities for this condition in our country as we get ready to offer newborn screening for this condition.

Funding: None. Competing interests: None stated.

\section{REFERENCES}

1. Kabra SK, Kabra M, Lodha R, Shastri S, Ghosh M, Pandey RM, et al. Clinical profile and frequency of delta f508 mutation in Indian children with cystic fibrosis. Indian Pediatr. 2003;40:612-9.

2. Ashavaid TF, Raghavan R, Dhairyawan P, Bhawalkar S. Cystic fibrosis in India: a systematic review. J Assoc Physicians India. 2012;60:39-41.

3. Kabra M, Ghosh M, Kabra SK, Khanna A, Verma IC. Delta F 508 molecular mutation in Indian children with cystic fibrosis. Indian J Med Res. 1996;104:355-8.

4. Sachdeva K, Saxena R, Puri R, Bijarnia S, Kohli S, Verma IC. Mutation analysis of the CFTR gene in 225 children: identification of five novel severe and seven reported severe mutations. Genet Test Mol Biomarkers. 2012;16:798-801.

5. Sharma N, Singh M, Kaur G, Thapa BR, Prasad R. 